\title{
Stochastic Resonance in the Majority Vote Model on Scale-Free Networks
}

\author{
A. KRAWIECKI* AND R.A. KosińsKi \\ Faculty of Physics, Warsaw University of Technology, \\ Koszykowa 75, 00-662 Warsaw, Poland
}

Received: 11.06.2020 \& Accepted: 04.09.2020

Doi: 10.12693/APhysPolA.138.824

*e-mail: andrzej.krawiecki@pw.edu.pl

\begin{abstract}
Stochastic resonance in the majority vote model on scale-free networks, exposed to a weak periodic signal, is investigated by means of Monte Carlo simulations and theoretically using heterogeneous mean field approximation and linear response theory. In numerical simulations, spectral power amplification shows a maximum as a function of the intensity of internal noise for a broad range of parameters of the model which confirms the occurrence of stochastic resonance. For the model on weakly heterogeneous or heterogeneous uncorrelated scale-free networks in the adiabatic limit of slowly varying periodic signal, a good quantitative agreement is obtained between predictions of the mean field approximation and results of Monte Carlo simulations. For the model on scale-free networks with fully developed heterogeneity, the occurrence of structural stochastic multiresonance characterized by double maxima of the spectral power amplification at different values of the intensity of internal noise is predicted theoretically which is not observed in Monte Carlo simulations.
\end{abstract}

topics: stochastic resonance, complex networks, majority vote model

\section{Introduction}

The majority vote (MV) model $[1,2]$ is a stochastic model of opinion formation in which agents with two opinions on a given subject - represented by two-state spins - update their opinions at discrete time steps. The opinion evolution is influenced by the opinion of the majority of their neighbors with certain probability $(1-q)$, where $0 \leq q \leq 1 / 2$ and $q$ is the parameter controlling intensity of internal noise. The MV model is a nonequilibrium counterpart of the Ising model for which an update rule for the spins depends only on the state of the system in their neighborhood (there is no global Hamiltonian) and does not obey the detailed balance condition. It was shown that the MV model on regular $d$-dimensional lattices $d=2,3,4 \ldots[1,3-5]$ as well as on different complex networks [6-14] exhibits a second-order phase transition from a disordered (paramagnetic, PM) state to an ordered (ferromagnetic, FM) state at $0<q_{c}<1 / 2$. In fact, much effort was made to determine the universality class for this transition. Interestingly, it has recently been shown that a first-order FM transition can occur in the MV model with agents endowed with certain forms of inertia $[15,16]$.

The critical properties of the MV model are relatively well understood, however, much less is known about the effect of a periodic signal on this model (in the form of an external bias of the majority opinion). For example, it was shown that a response of the MV model on regular lattices to such a stimulation can exhibit maximum periodicity at a non-zero level of the internal noise $q$ [17]. This result is an example of stochastic resonance (SR), a phenomenon in which a (usually nonlinear) system stimulated by a weak periodic signal shows a maximally periodic behavior for non-zero intensity of external or internal noise [18-20]. On the other hand, in the Ising model, SR consisting in the maximization of the periodicity of the response of the system to a weak oscillating magnetic field at a non-zero temperature was reported in a broad variety of cases, e.g., in the model on regular lattices [21-25] and on different complex networks [26-28]. The investigation of SR in systems on regular and complex networks is an important topic in statistical physics [29-31]. Thus, in this paper, the occurrence of SR in the MV model with an external periodic signal on heterogeneous complex scale-free (SF) networks $[32,33]$ is demonstrated by means of Monte Carlo (MC) simulations and the numerical results are compared to analytical predictions obtained using the heterogeneous mean-field approximation (MFA) and the linear response theory (LRT).

Stochastic multiresonance (SMR) is a type of SR in which the periodicity of the response of a nonlinear system to a weak oscillating signal is maximized for several intensities of the external or internal noise [34, 35]. SMR was observed, e.g., in systems of many interacting units such as neurons [36-39], threshold elements [40], spins in the Ising model [28] 
and - for narrow intervals of the amplitude of the oscillating signal - in the MV model on regular and small-world lattices [17]. In particular, the so-called structural SMR was reported in the Ising model on certain SF networks [28]. Its occurrence is related to strong heterogeneity of SF networks leading to the divergence of the critical temperature for the FM transition in the thermodynamic limit [41]. Structural SMR consists in the maximization of the periodicity of the response of the model with a large, although finite number of spins to a weak oscillating magnetic field for at least two different temperatures. In this paper, it is shown that under similar assumptions concerning the degree of heterogeneity of SF networks, the analytical calculations based on the MFA and LRT predict the occurrence of SMR in the MV model. According to these predictions, the response of the appropriate MV model with a large number of spins to a periodic signal should exhibit maximum periodicity for two different non-zero values of the parameter $q$ characterizing the level of internal noise. However, in contrast to the case of the Ising model on SF networks, MC simulations of the MV model do not confirm the appearance of SMR.

\section{Model}

\subsection{Majority vote model with external periodic signal on complex networks}

The model under study and the method of characterization of SR in this model is introduced in this section. The MV model without an external signal consists of agents represented by two-state spins $s_{i}= \pm 1, i=1,2, \ldots N$ located in the nodes of a network of interactions which change their opinions taking into account the opinion of the majority of their neighbors. The probability per unit time (rate) that the spin $s_{i}$ in node $i$ flips is

$$
w_{i}(\boldsymbol{s})=\frac{1}{2}\left(1-(1-2 q) s_{i} \operatorname{sgn}_{i}\right),
$$

where $s$ denotes the spin configuration and $q$ is the model parameter controlling the intensity of internal noise. The signum function $\operatorname{sgn}_{i}$ is defined as

$$
\operatorname{sgn}_{i}=\operatorname{sgn}\left(\sum_{j \in n n_{i}} s_{j}\right)
$$

where

$$
\operatorname{sgn}(x)= \begin{cases}-1 & \text { for } x<0 \\ 0 & \text { for } x=0 \\ +1 & \text { for } x>0\end{cases}
$$

and where $n n_{i}$ denotes a set of the nearest neighbors of the node $i$, i.e., that of nodes connected directly by an edge to the node $i$. In this way, the agents follow the opinion of the majority of their neighbors with the probability $(1-q)$ or the opposite opinion with the probability $q$. The sets of the nearest neighbors for the consecutive nodes depend on the network topology. Here, the studied MV model on complex heterogeneous networks is characterized by the degree distribution $p(k)$ (distribution of the number of edges $k_{i}$ attached to the nodes $i=1,2, \ldots N)$. In particular, the case of SF networks with $p(k) \propto k^{-\gamma}$, with $\gamma>2[32,33]$ is investigated.

In this paper, we consider the effect of a weak periodic signal of the amplitude $A \ll 1$ in the form of an external bias of the majority opinion on the behavior of the MV model. It results in the periodic time-dependence of the spin flip rate (1) via

$$
\operatorname{sgn}_{i}=\operatorname{sgn}\left(\sum_{j \in n n_{i}} s_{j}+A \sin \left(\omega_{0} t\right)\right) .
$$

The response of the MV model to this form of periodic stimulation is studied as a function of the internal noise parameter $q$ for different frequencies $\omega_{0}$ and different degree distributions $p(k)$. The response signal is assumed as the time-dependent weighted magnetization $S(t)=\frac{1}{N\langle k\rangle} \sum_{i=1}^{N} k_{i} s_{i}(t)$, where $\langle k\rangle$ is the mean degree of nodes and $t$ denotes the discrete MC simulation steps. Such weighted magnetization is a typical order parameter in systems on heterogeneous networks and was used, e.g., in the investigation of SR in the Ising model on SF networks [28]. It is verified that $\mathrm{SR}$ can be observed in the MV model as the level of internal noise $q$ is varied. For this purpose, one of typical measures of $\mathrm{SR}$, called the spectral power amplification (SPA), is investigated as a function of $q$, defined as:

$$
\begin{aligned}
& \mathrm{SPA}=\frac{\left|P_{1}\right|^{2}}{A^{2}}, \\
& P_{1}=\frac{1}{T_{0}} \int_{0}^{T_{0}} S(t) \mathrm{e}^{\mathrm{i} \omega_{0} t} \mathrm{~d} t,
\end{aligned}
$$

where $T_{0}=2 \pi / \omega_{0}$ is the period of the oscillating signal and $P_{1}$ is the corresponding Fourier component of the response signal. In the systems with $\mathrm{SR}$, the SPA should show a maximum at a non-zero noise level. In the case of the MV model, the SPA vs. $q$ shows a maximum at a non-zero level of the internal noise $q=q_{\max }>0$.

It should be mentioned that the assumption $A \ll 1$, meaning weak periodic signal, is typical in the studies of SR but not necessary. It is used in most systems exhibiting SR since it facilitates theoretical analysis which can be then performed on the basis of the LRT $[19,20]$. In the case of the MV model studied in this paper, defined by (1) and (4), the applicability of the LRT is limited by the model's threshold character which leads to the situation when even an infinitesimally weak signal with $A \rightarrow 0$ can cause a finite response in the weighted magnetization $S(t)$ (see Sect. 4). Nevertheless, the assumption $A \ll 1$ is kept here since it enables a direct comparison of the properties of SR in the MV model on complex networks with those in the corresponding Ising model which were obtained under the assumption of a weak oscillating magnetic field [26-28]. Relaxing this assumption can lead, 
e.g., to the occurrence of SMR for the amplitudes $A$ close to natural numbers, as in the case of the MV on regular networks [17]. However, this sort of SMR is not structural SMR since it results from the form of the spin flip rate (4), containing the signum function, rather than from the topology of the network of interactions, hence, it is not investigated in this paper.

\subsection{Models for scale-free networks}

SF networks are characterized by the degree distribution $p(k)=0$ for $k<k_{\min }$ and by $p(k)=C k^{-\gamma}$ for $k_{\min } \leq k \leq k_{\max }$, where $k_{\min }$ and $k_{\max }$ are minimum and maximum degrees of nodes, and $C$ is the normalization constant. The moments of order $\nu$ of the degree distribution are thus

$$
\left\langle k^{\nu}\right\rangle=\int_{k_{\min }}^{k_{\max }} p(k) k^{\nu} \mathrm{d} k .
$$

For the purpose of investigation of SR in the model under study, it is convenient to distinguish between SF networks with the number of nodes $N \rightarrow \infty$ (the thermodynamic limit) and SF networks with a large but finite $N$. It is believed that the occurrence of SMR might be related to finite-size effects. In the thermodynamic limit, it is fulfilled that $k_{\max } \rightarrow \infty$, thus $C=(\gamma-1)\left(k_{\min }\right)^{\gamma-1}$. The mean degree of nodes, i.e., the first moment of the degree distribution $\langle k\rangle$ is finite for $\gamma>2$ which imposes a lower limit on the possible values of the exponent $\gamma$. Even if $\langle k\rangle$ is finite, higher-order moments of the degree distribution $\left\langle k^{\nu}\right\rangle, \nu>1$, diverge for $\gamma<\nu+1$. In particular, for the study of many models on SF networks, including the Ising model, it is important that the second moment of the degree distribution $\left\langle k^{2}\right\rangle \rightarrow \infty$ for $2<\gamma \leq 3[33,41]$. In the case of networks with a finite number of nodes $N$, the maximum degree of nodes $k_{\max }=k_{\max }(N)$ is of course finite - it depends on the method of generation of the SF network and in general increases as a power function of $N$. Thus, for large $k_{\max }$ (large $N)$, there is still $C \approx(\gamma-1)\left(k_{\min }\right)^{\gamma-1}$ and all moments of the degree distribution are finite.

A simple and efficient way to generate SF networks with given degree distributions is to use the configuration model (CM) [42]. This algorithm starts with assigning to each node $i$ - in a set of $N$ nodes - a degree, i.e., a random number $k_{i}$ of ends of edges drawn from a given probability distribution $p(k)$, with $k_{\min }<k_{i}<N$ and with the condition that the sum $\sum_{i=1}^{N} k_{i}$ is even. The network is completed by connecting pairs of the ends of edges chosen uniformly at random to make complete edges, respecting the preassigned sequence $k_{i}$ and under the condition that multiple and selfconnections are forbidden. In SF networks generated from the CM for large but finite $N$, the maximum degree in practice scales as $k_{\max } \approx k_{\min } \sqrt{N}$ for $\gamma>3$ and $k_{\max } \approx k_{\min } N^{1 /(\gamma-1)}$ for $2<\gamma \leq 3$.
For $\gamma \geq 3$, the SF networks obtained from the CM are weakly heterogeneous, i.e., they do not contain many hubs (nodes with very large degrees) and simultaneously are uncorrelated (the correlation between degrees of nodes connected directly by edges is negligible). In contrast, for $2<\gamma<3$, these networks are strongly heterogeneous and correlated (disassortative) since nodes with high degrees are more probably connected by edges to nodes with small degrees. In order to avoid such correlations, the networks can be generated from the uncorrelated configuration model (UCM) [43]. The only difference between the UCM model and the CM model is that the degrees $k_{i}$, assigned randomly to the nodes, are limited to the interval $k_{\min }<k_{i}<\sqrt{N}$. The SF networks generated from the UCM are uncorrelated for $\gamma>2$ but their heterogeneity is not fully developed since the maximum degree scales as $k_{\max } \approx \sqrt{N}$ for $\gamma>2$.

In the Ising model on SF networks, SMR was observed only in the case of strongly heterogeneous networks with $2<\gamma<3$ generated from the CM. It is a finite-size effect related to a strong dependence of the critical temperature for the FM transition on the number of spins $N$. SMR was not observed in the case of weakly heterogeneous networks, i.e., networks with $\gamma \geq 3$ generated from the $\mathrm{CM}$ or networks with $\gamma>2$ generated from the UCM [28]. Therefore, in this paper, SR in the non-equilibrium counterpart of the Ising model, the MV model on SF networks with different exponents $\gamma>2$ in the degree distributions generated from the CM and UCM models, is investigated. The objective of this investigation is to verify whether and under what conditions SMR occurs.

\section{Heterogeneous mean field approximation}

In this section, we derived the time-dependent equation of the weighted magnetization of the periodically driven MV model in the MFA by taking into account the heterogeneous structure of the underlying SF networks. A starting point is the master equation for a general spin system in which dynamics is governed by the transition rates $w\left(\boldsymbol{s} \mid \boldsymbol{s}^{\prime}\right)$ from the spin configuration $\boldsymbol{s}^{\prime}$ to $\boldsymbol{s}$. The probability $P(s, t)$ that at the time $t$, the spin configuration is $s$ obeys

$$
\begin{aligned}
& \frac{\mathrm{d} P(\boldsymbol{s}, t)}{\mathrm{d} t}= \\
& \sum_{\boldsymbol{s}^{\prime}}\left[w\left(\boldsymbol{s} \mid \boldsymbol{s}^{\prime}\right) P\left(\boldsymbol{s}^{\prime}, t\right)-w\left(\boldsymbol{s}^{\prime} \mid \boldsymbol{s}\right) P(\boldsymbol{s}, t)\right] .
\end{aligned}
$$

We take into account that in the MV under study at each time step a transition occurs between spin configurations $s^{\prime} \rightarrow s$ differing just by one spin flipped, say $s_{i}$. The transition rate $w\left(\boldsymbol{s} \mid \boldsymbol{s}^{\prime}\right)=w_{i}\left(\boldsymbol{s}^{\prime}\right)$ is given by (1). By performing the ensemble average of (8), the following equation is obtained: 


$$
\frac{\partial\left\langle s_{i}\right\rangle}{\partial t}=-2\left\langle s_{i} w_{i}(\boldsymbol{s})\right\rangle=-\left\langle s_{i}\right\rangle+(1-2 q)\left\langle\operatorname{sgn}_{i}\right\rangle
$$

where $\left\langle s_{i}\right\rangle$ denotes the mean value of spin in node $i$ and

$$
\begin{gathered}
\left\langle\operatorname{sgn}_{i}\right\rangle=(+1) \operatorname{Pr}\left(\operatorname{sgn}_{i}=+1\right) \\
+(-1) \operatorname{Pr}\left(\operatorname{sgn}_{i}=-1\right) .
\end{gathered}
$$

Let us consider the MV model on a general heterogeneous network, where the nodes are characterized by their degrees $k_{i}$ which are drawn from a given probability distribution $p(k)$. The minimum degree of nodes is $k_{\min }$. The maximum degree $k_{\max }$ is a function of the number of nodes $N$, as explained in Sect. 2.2, and is finite for finite $N$, and in general diverges to infinity in the thermodynamic limit $(N \rightarrow \infty)$. For a weak periodic signal with the amplitude $A \ll 1$, the signum function (4) is equal to $+1(-1)$ if the majority of spins in the neighborhood of the node $i$ has the orientation up (down) or if the numbers of spins with opposite orientations are equal, while the periodic signal should have a positive (negative) value. The latter situation is possible only if the number of neighbors of the $i$ node, i.e., the degree $k_{i}$, is even. Assuming that orientations of different spins belonging to the neighborhood of the node $i$ are independent twostate random variables, it follows that

$$
\begin{aligned}
& \operatorname{Pr}\left(\operatorname{sgn}_{i}= \pm 1\right)= \\
& \quad \sum_{l=\left\lceil\frac{k_{i}}{2}\right\rceil}^{k_{i}}\left(\begin{array}{c}
k_{i} \\
l
\end{array}\right) \prod_{j}^{l} \operatorname{Pr}\left(s_{j}= \pm 1\right) \prod_{j \prime}^{k_{i}-l} \operatorname{Pr}\left(s_{j^{\prime}}=\mp 1\right) \\
& +\frac{\delta_{k_{i}, \text { even }}}{2}\left(1 \pm \operatorname{sgn}\left(A \sin \left(\omega_{0} t\right)\right)\right)\left(\begin{array}{c}
k_{i} \\
\frac{k_{i}}{2}
\end{array}\right) \\
& \times \prod_{j}^{k_{i} / 2} \operatorname{Pr}\left(s_{j}=+1\right) \prod_{j^{\prime}}^{k_{i} / 2} \operatorname{Pr}\left(s_{j^{\prime}}=-1\right), \quad(11)
\end{aligned}
$$

where $\lceil\cdot\rceil$ denotes the ceil function, $j, j^{\prime}$ denote the nodes connected with the node $i$ by edges and

$$
\delta_{k_{i}, \text { even }}=\left\{\begin{array}{l}
0 \text { for } k=1,3,5 \ldots \\
1 \text { for } k=2,4,6 \ldots
\end{array}\right.
$$

In order to evaluate the probabilities in (11), the heterogeneous MFA will be applied. The main assumption for this approximation is that the nodes can be divided into classes according to their degrees and that spins in the nodes belonging to the same class are equivalent. Then for the spin $s_{i}$ located in the node with degree $k_{i}$ there is

$$
\operatorname{Pr}\left(s_{i}= \pm 1\right)=\frac{1 \pm\left\langle s_{i}\right\rangle}{2}=\frac{1 \pm\left\langle s_{k_{i}}\right\rangle}{2},
$$

where $\left\langle s_{k_{i}}\right\rangle$ denotes the mean value of the spin in each node with degree $k_{i}$.

Progress in analytical considerations can be achieved in the case of uncorrelated networks. In such networks, the probability that an edge attached to the node $i$ points at the other end at the node with degree $k$ does not depend on $k_{i}$ and is $k p(k) /\langle k\rangle$. Thus, among the $l$ nodes in the first product in (11), containing spins with the same orientation as that of the spin in the node $i$, formally there are $l k p(k) /\langle k\rangle$ nodes which belong to a given class of nodes with degree $k$. Similarly, among $\left(k_{i}-l\right)$ nodes in the second product in (11) - containing spins with the opposite orientation than that of the spin in the node $i$ - formally there are $\left(k_{i}-l\right) k p(k) /\langle k\rangle$ nodes belonging to a class of nodes with degree $k$, etc. As a result, products over the indices of nodes $j, j^{\prime}$ in (11) can be replaced with the product over the degrees (classes) of nodes $k$. Now, using (13), the probabilities in (11) can be written as

$$
\begin{aligned}
& \operatorname{Pr}\left(\operatorname{sgn}_{i}= \pm 1\right)=\sum_{l=\left\lceil\frac{k_{i}}{2}\right.} k_{i}\left(\begin{array}{c}
k_{i} \\
l
\end{array}\right) \\
& \times \prod_{k=k_{\min }}^{k_{\max }}\left(\frac{1 \pm\left\langle s_{k}\right\rangle}{2}\right)^{l \frac{k p(k)}{\langle k\rangle}}\left(\frac{1 \mp\left\langle s_{k}\right\rangle}{2}\right)^{\left(k_{i}-l\right) \frac{k p(k)}{\langle k\rangle}} \\
& +\frac{\delta_{k_{i}, \text { even }}}{2}\left(1 \pm \operatorname{sgn}\left(A \sin \left(\omega_{0} t\right)\right)\right)\left(\begin{array}{c}
k_{i} \\
\frac{k_{i}}{2}
\end{array}\right) \\
& \times \prod_{k=k_{\min }}^{k_{\max }}\left(\frac{1-\left\langle s_{k}\right\rangle^{2}}{4}\right)^{\frac{k_{i}}{2} \frac{k p(k)}{\langle k\rangle}} .
\end{aligned}
$$

Approximating $\left(1 \pm\left\langle s_{k}\right\rangle\right)^{\frac{k p(k)}{\langle k\rangle}} \approx 1 \pm \frac{k p(k)}{\langle k\rangle}\left\langle s_{k}\right\rangle$ and introducing the weighted magnetization as the order parameter,

$$
S=\frac{1}{N\langle k\rangle} \sum_{i=1}^{N} k_{i}\left\langle s_{i}\right\rangle=\sum_{k=k_{\min }}^{k_{\max }} \frac{k p(k)}{\langle k\rangle}\left\langle s_{k}\right\rangle,
$$

where, again, the summation over the indices of nodes in the first sum of (15) is replaced with the sum over the degrees (classes) of nodes $k$, it is obtained that

$$
\begin{aligned}
& \operatorname{Pr}\left(\operatorname{sgn}_{i}= \pm 1\right)= \\
& \sum_{l=\left\lceil\frac{k_{i}}{2}\right\rceil}^{k_{i}}\left(\begin{array}{c}
k_{i} \\
l
\end{array}\right)\left(\frac{1 \pm S}{2}\right)^{l}\left(\frac{1 \mp S}{2}\right)^{k_{i}-l} \\
& +\frac{\delta_{k_{i}, \text { even }}}{2}\left(1 \pm \operatorname{sgn}\left(A \sin \left(\omega_{0} t\right)\right)\right) \\
& \quad \times\left(\begin{array}{c}
k_{i} \\
\frac{k_{i}}{2}
\end{array}\right)\left(\frac{1-S^{2}}{4}\right)^{\frac{k_{i}}{2}} .
\end{aligned}
$$

For large $k_{i}$, the binomial distribution in the first term can be approximated by the normal distribution while in the second term the Stirling approximation

$$
2^{-k_{i}}\left(\begin{array}{l}
k_{i} \\
\frac{k_{i}}{2}
\end{array}\right) \approx \frac{2}{\sqrt{2 \pi k_{i}}}
$$

can be made. Thus, 


$$
\begin{aligned}
& \operatorname{Pr}\left(\operatorname{sgn}_{i}= \pm 1\right) \approx \frac{1}{2} \pm \frac{1}{2} \operatorname{erf}\left(\sqrt{\frac{k_{i}}{2}} \frac{S}{\sqrt{1-S^{2}}}\right) \\
& \quad+\delta_{k_{i}, \text { even }}\left(1 \pm \operatorname{sgn}\left(A \sin \left(\omega_{0} t\right)\right)\right) \frac{\left(1-S^{2}\right)^{k_{i} / 2}}{\sqrt{2 \pi k_{i}}}
\end{aligned}
$$

Substituting (18) into (10) and then into (9), it turns out that for spins located in nodes with large degrees one has

$$
\begin{gathered}
\frac{\partial\left\langle s_{i}\right\rangle}{\partial t}=-\left\langle s_{i}\right\rangle+(1-2 q) \operatorname{erf}\left(\sqrt{\frac{k_{i}}{2}} \frac{S}{\sqrt{1-S^{2}}}\right) \\
+\frac{2(1-2 q)\left(1-S^{2}\right)^{\frac{k_{i}}{2}}}{\sqrt{2 \pi k_{i}}} \operatorname{sgn}\left(A \sin \left(\omega_{0} t\right)\right) \delta_{k_{i}, \text { even }} .
\end{gathered}
$$

Multiplying now both sides of (19) by $k_{i}$, performing summation over all nodes and, next, replacing it on the right-hand side by the sum over the degrees (classes) of nodes, the MF equation for the timedependence of the order parameter is obtained:

$$
\begin{aligned}
& \frac{\partial S}{\partial t}=(1-2 q) \sum_{k=k_{\min }}^{k_{\max }} \frac{k p(k)}{\langle k\rangle} \operatorname{erf}\left(\sqrt{\frac{k}{2}} \frac{S}{\sqrt{1-S^{2}}}\right) \\
& -S+\frac{(1-2 q)}{\sqrt{2 \pi}} \sum_{k=k_{\min }}^{k_{\max }} \frac{p(k) \sqrt{k}\left(1-S^{2}\right)^{k / 2}}{\langle k\rangle} \\
& \quad \times \operatorname{sgn}\left(A \sin \left(\omega_{0} t\right)\right) .
\end{aligned}
$$

In the last term, which describes the effect of the periodic signal, the sum taken over classes of nodes with only even degrees is approximated by half of sum over all classes of nodes. In such a case, the factor 2 present in (19) now disappears.

In general, (20) should provide a good approximation of the time-dependent order parameter $S$ in the MV model on uncorrelated networks with large degrees of nodes. For SF networks under study, these conditions mean that $\gamma>3(\gamma>2)$ in the case of networks generated from the CM (UCM) and that $k_{\text {min }}$ is large enough. However, some remarks on the validity of the MF approach in the model under study are necessary.

Firstly, the MF approximation in (9) well describes the relaxation of the order parameter toward its equilibrium value but does not take into account its fluctuations. Hence, the MFA yields more correct results in adiabatic limit $\omega_{0} \rightarrow 0$ of a slowly varying periodic signal when the model remains in a quasi-equilibrium regime. Secondly, the introduction of the order parameter $S$ in (15) is possible only because of partial linearization of (14). As a result, (20) becomes more correct if the model remains in the vicinity of the $\mathrm{PM}$ state with all $\left\langle s_{i}\right\rangle \approx 0$ and $S \approx 0$. The mentioned limitations of the MF approach are, in some cases, a source of discrepancies between the results of simulations of (20) and those of direct $\mathrm{MC}$ simulations of the corresponding MV model reported in Sect. 5 .
The absence of the periodic signal $(A=0)$ causes that (20) possesses stationary solutions $S_{0}$ of the form

$$
S_{0}=(1-2 q) \sum_{k=k_{\min }}^{k_{\max }} \frac{k p(k)}{\langle k\rangle} \operatorname{erf}\left(\sqrt{\frac{k}{2}} \frac{S_{0}}{\sqrt{1-S_{0}^{2}}}\right) .
$$

This solution fulfils the condition $\mathrm{d} S / \mathrm{d} t=0$. Moreover, one can show that in the thermodynamic limit, when $N \rightarrow \infty$, the stable solution of $(21)$ is $S_{0}=0$ for $q>q_{c}$ (corresponding to the disordered (PM) phase), while for $q<q_{c}$ there are two symmetric stable solutions: $\pm S_{0}$ and $S_{0}>0$. The latter solutions correspond to the ordered (FM) phase. The critical value of the internal noise for the FM transition [11] is given as

$$
q_{c}=\frac{1}{2}-\frac{\sqrt{2 \pi}}{4} \frac{\langle k\rangle}{\left\langle k^{3 / 2}\right\rangle}
$$

In the case of SF networks with $\gamma>5 / 2$, the MFA predicts the FM type of transition at $q_{c}=\frac{1}{2}-\frac{\sqrt{2 \pi}}{4} \frac{\gamma-5 / 2}{\gamma-2}\left(k_{\min }\right)^{-1 / 2}$ in the MV model. On the other hand, for $2<\gamma<5 / 2$ the moment $\left\langle k^{3 / 2}\right\rangle$ is infinite and it turns out that $q_{c}=1 / 2$. This only means that the FM phase is stable for any level of the internal noise. This also corresponds to the divergence of the critical temperature for the Ising model on SF networks with $2<\gamma<3$ [41].

Note that the MFA is valid only in the thermodynamic limit, however, it can also provide valuable estimates for the critical behavior of the model with a large but finite number of $N$ nodes. In this case, FM ordering with $S_{0}>0$ occurs below a crossover value $q_{c}^{*}=q_{c}^{*}(N)$ - still given by $(22)$. In fact, the condition $q_{c}^{*}<1 / 2$ is always valid, and

$q_{c}^{*}(N) \stackrel{N \rightarrow \infty}{\longrightarrow} \begin{cases}q_{c}, & \text { for } \gamma>\frac{5}{2} \\ \frac{1}{2}, & \text { for } 2<\gamma<\frac{5}{2}, \text { with } \\ 1-2 q_{c}^{*}(N) \propto\left(k_{\max }(N)\right)^{\gamma-\frac{5}{2}}\end{cases}$

Taking into account the scaling of the maximum degree with $N$ (Sect. 2.2) in the case of SF networks generated from the CM model, one has that $\left(1-2 q_{c}^{*}(N)\right) \propto N^{(\gamma-5 / 2) /(\gamma-1)}$, i.e., $q_{c}^{*}(N)$ converges fast to $q_{c}=1 / 2$, while in the case of $\mathrm{SF}$ networks generated from the UCM model one has that $\left(1-2 q_{c}^{*}(N)\right) \propto N^{(\gamma-5 / 2) / 2}$, i.e., $q_{c}^{*}(N)$ converges more slowly to $q_{c}=1 / 2$.

\section{Linear response theory}

In the presence of a weak periodic signal, where $A \ll 1$, an approximate solution of $(20)$ can be obtained in the framework of the LRT. The last term of (20) is treated as a small perturbation and so the solution is assumed in the form of small oscillations around the fixed point corresponding to a given value of $q$. Then one has (i) $S_{0}>0$ for $q<q_{c}$ in the thermodynamic limit (for $q<q_{c}^{*}(N)$ in the case of SF network with a finite number of 
nodes $N$ ) and (ii) $S_{0}=0$ for $q>q_{c}\left(q>q_{c}^{*}(N)\right)$. Now, assuming that $S=S_{0}+\xi$ and further expanding (20) up to linear terms in $\xi$, one obtains

$$
\frac{\mathrm{d} \xi}{\mathrm{d} t}=-\frac{\xi}{\tau}+\alpha(t)
$$

This is a linear differential equation for the timedependence of $\xi$ which converges exponentially with characteristic damping time $\tau$ :

$$
\begin{aligned}
\tau^{-1} & =1-\frac{2}{\sqrt{2 \pi}} \frac{1-2 q}{\left(1-S_{0}^{2}\right)^{3 / 2}} \\
& \times \sum_{k=k_{\min }}^{k_{\max }} \frac{k^{3 / 2} p(k)}{\langle k\rangle} \exp \left(-\frac{k S_{0}^{2}}{2-2 S_{0}^{2}}\right)
\end{aligned}
$$

to an asymptotic oscillating solution determined by the external perturbation

$$
\begin{gathered}
\alpha(t)=\frac{(1-2 q)}{\sqrt{2 \pi}} \sum_{k=k_{\min }}^{k_{\max }} \frac{k^{1 / 2} p(k)}{\langle k\rangle} \\
\times\left(1-S_{0}^{2}\right)^{k / 2} \operatorname{sgn}\left(A \sin \left(\omega_{0} t\right)\right) .
\end{gathered}
$$

Now, expanding $\alpha(t)$ in the Fourier series, one yields

$$
\begin{aligned}
& \alpha(t)=\sum_{j=1}^{\infty} \alpha_{j} \sin \left(j \omega_{0} t\right), \\
& \alpha_{j}= \\
& \begin{cases}\frac{4}{j \pi} \frac{1-2 q}{\sqrt{2 \pi}} \sum_{k} \frac{k^{1 / 2} p(k)\left(1-S_{0}^{2}\right)^{k / 2}}{\langle k\rangle} & \text { for } j=1,3,5 \ldots \\
0 & \text { for } j=2,4,6 \ldots\end{cases}
\end{aligned}
$$

For $q>q_{c}\left(q>q_{c}^{*}(N)\right)$, there is $S_{0}=0$ in the PM phase and

$$
\tau^{-1}=1-\frac{2(1-2 q)}{\sqrt{2 \pi}} \frac{\left\langle k^{3 / 2}\right\rangle}{\langle k\rangle} .
$$

Note that $\tau^{-1}=0$ for $q=q_{c}\left(q=q_{c}^{*}(N)\right)$, as expected, and also $\tau^{-1} \rightarrow 1$ as $q \rightarrow 1 / 2$. The latter means that for $q>q_{c}$ the damping time $\tau$ is a decreasing function of $q$ and

$$
\alpha_{j}=\left\{\begin{array}{cl}
\frac{4}{j \pi} \frac{1-2 q}{\sqrt{2 \pi}} \frac{\left\langle k^{1 / 2}\right\rangle}{\langle k\rangle} & \text { for } j=1,3,5 \ldots \\
0 & \text { for } j=2,4,6 \ldots
\end{array}\right.
$$

The asymptotic solution of (24) when $t \rightarrow \infty$ is

$$
\xi(t)=\sum_{j=1}^{\infty} \frac{\alpha_{j}}{\sqrt{\tau^{-2}+\left(j \omega_{0}\right)^{2}}} \sin \left(j \omega_{0} t-\phi_{j}\right),
$$

where $\phi_{j}=\tan ^{-1}\left(j \omega_{0} \tau\right)$. With the use of (5), (6) and (31), the SPA can be expressed as

$$
\mathrm{SPA}=\frac{1}{4 A^{2}} \frac{\left|\alpha_{1}\right|^{2}}{\tau^{-2}+\omega_{0}^{2}} .
$$

In particular, at $q=q_{c}\left(q=q_{c}^{*}(N)\right)$,

$$
\left.\mathrm{SPA}\right|_{q=q_{c}, q=q_{c}^{*}(N)}=\frac{1}{A^{2} \pi^{2} \omega_{0}^{2}}\left(\frac{\left\langle k^{1 / 2}\right\rangle}{\left\langle k^{3 / 2}\right\rangle}\right)^{2} .
$$

Note that for the model on SF networks in the thermodynamic limit, (33) predicts

$$
\left.\mathrm{SPA}\right|_{q_{c}}= \begin{cases}\frac{\left(\gamma-\frac{5}{2}\right)}{\left(A \pi^{2} \omega_{0}^{2} k_{\min }\right)^{2}\left(\gamma-\frac{3}{2}\right)}, & \text { for } \gamma>\frac{5}{2} \\ 0, & \text { for } 2<\gamma<\frac{5}{2} \\ & \text { when }\left\langle k^{3 / 2}\right\rangle \rightarrow \infty\end{cases}
$$

In the case $\gamma>5 / 2$ and for $q>q_{c}$, the SPA decreases to zero as $q \rightarrow 1 / 2$. For the model on SF networks with a large but finite $N,\left.\mathrm{SPA}\right|_{q=q_{c}^{*}(N)}$ is always finite and also for $q>q_{c}^{*}(N)$ it decreases to zero as $q \rightarrow 1 / 2$. For $\gamma>\frac{5}{2}$ there is $\left.\left.\mathrm{SPA}\right|_{q=q_{c}^{*}(N)} \stackrel{N \rightarrow \infty}{\longrightarrow} \mathrm{SPA}\right|_{q=q_{c}}$, while for $2<\gamma<\frac{5}{2}$, one has $\left.\mathrm{SPA}\right|_{q=q_{c}^{*}(N)} \propto\left(k_{\max }\right)^{2 \gamma-5}$. The latter is also

$\left.\mathrm{SPA}\right|_{q=q_{c}^{*}(N)} \propto \begin{cases}N^{\frac{2 \gamma-5}{\gamma-1}} \stackrel{N \rightarrow \infty}{\longrightarrow} 0 & \text { from the CM, } \\ N^{\gamma-5 / 2} \stackrel{N \rightarrow \infty}{\longrightarrow} 0 \text { from the UCM. }\end{cases}$

However, the above argument does not guarantee that at least a local maximum of the SPA is observed at $q=q_{c}\left(q=q_{c}^{*}(N)\right)$ since the SPA can, in principle, grow as $q$ decreases below the critical (crossover) value.

It should be noted that the LRT and (32) cannot predict the SPA correctly. This is because even for $A \rightarrow 0$ the $\alpha(t)$ perturbation in (24) remains finite. Since even an infinitesimally small periodic signal affects strongly the probability of the spin flip (4), thus the periodic term in (20) obviously remains finite for $A>0$. As a result and taking into account $(27)$ and (28), the response $\xi(t)$ also remains finite for any amplitude of the periodic signal. Moreover, $\xi(t)$ does not decrease to zero for $A \rightarrow 0$, as required when the LRT is applied. Hence, the SPA (32) diverges in the limit $A \rightarrow 0$ and no correct results are obtained with the LRT. A similar problem with the accuracy of the predictions of the LRT occurred also in the investigation of SR in the MV model on regular lattices [17]. This is in contrast with most systems in which SR is observed, including the Ising model on regular lattices [21-25] and complex networks [28].

\section{Results and discussion}

Theoretical predictions resulting from the heterogeneous MFA and LRT concerning SR in the MV model on weakly (with $\gamma=6$ ) and marginally (with $\gamma=3$ ) heterogeneous SF networks are summarized in Figs. 1 and 2, respectively, and compared with results of MC simulations. Predictions of the MFA were obtained by evaluating the SPA from (5) and (6) using the time-dependent order parameter $S(t)$ from numerical simulations of (20) and those of the LRT from (27)-(32). According to the MFA, in both cases in the absence of the periodic signal the model undergoes FM transition at $q_{c}<1 / 2$. In all cases for a broad range of the frequencies of the periodic signal $\omega_{0}$, all theoretical and numerical curves SPA vs. $q$ exhibit a maximum which is a signature of SR. The maxima of the SPA obtained from MC simulations and from simulations 


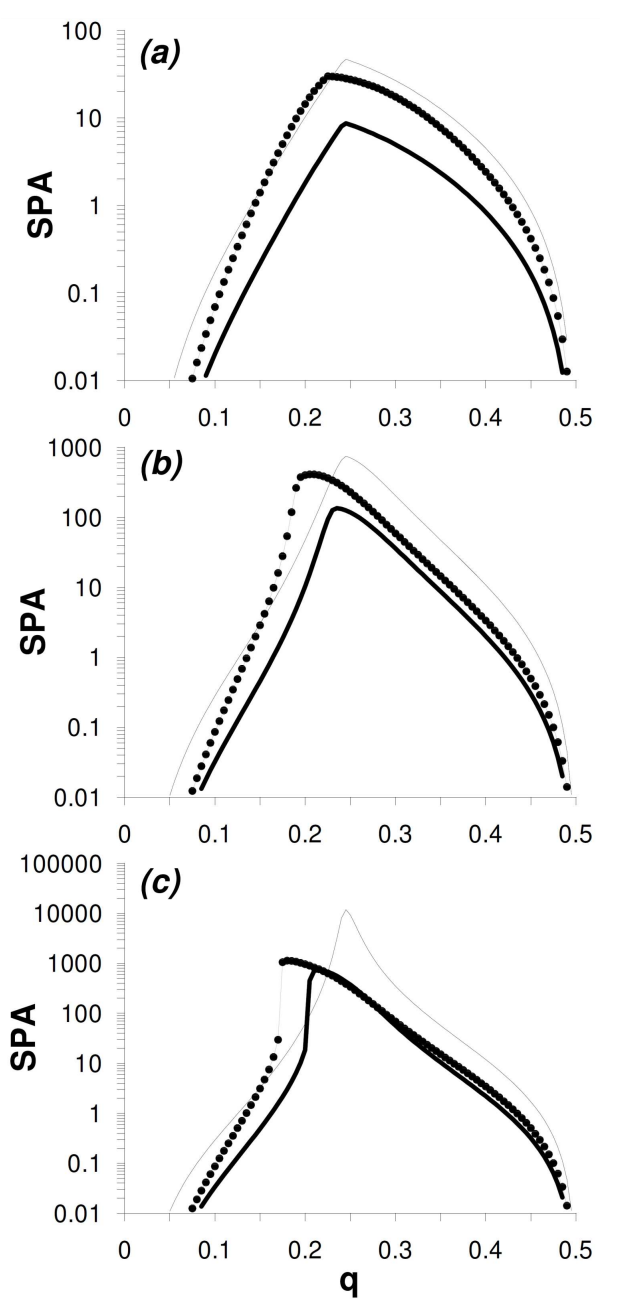

Fig. 1. SPA vs. $q$ for the MV model on an SF network obtained from the CM with $N=64000$, $k_{\min }=5$ and $\gamma=6$ for the periodic signal with $A=0.01$ and (a) $\omega_{0}=2 \pi / 8$, (b) $\omega_{0}=2 \pi / 32$, (c) $\omega_{0}=2 \pi / 128$. Symbols - results from MC simulations, gray solid lines are guides to the eyes, thick solid lines - results from numerical solution of the appropriate MF equations (20) and from (5) and (6), thin solid lines - results of the LRT, (27)-(32).

of the MF equations are located at $q$ slightly below $q_{c}$, although they are somewhat shifted with respect to each other, and those resulting from the LRT are located at $q \approx q_{c}$ which is best visible for small frequencies $\omega_{0}$. Qualitative agreement between theoretical curves SPA vs. $q$ and those from MC simulations is good in all cases and the best quantitative agreement is observed between predictions of the MFA from (20) and results of MC simulations in the adiabatic limit $\omega_{0} \rightarrow 0$ and for $q>q_{c}$, as expected (Figs. 1c and 2c). Quantitative agreement is better in the case of model on the SF network with $\gamma=6$ but is satisfactory also for $\gamma=3$, when the critical value $q_{c}$ for the MV model does not diverge with the number of nodes $N$, in contrast to the Ising model. On the other hand, in the adiabatic limit, the maxima of the SPA obtained using the LRT are much
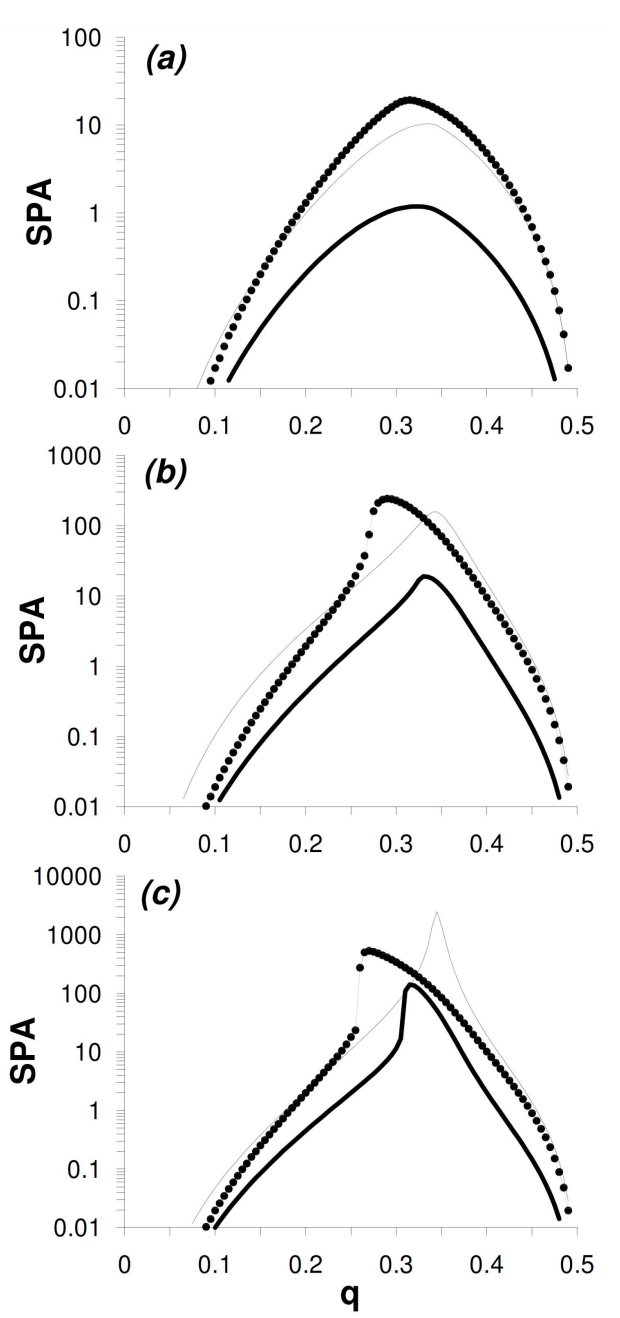

Fig. 2. As in Fig. 1 but for an SF network with $\gamma=3$.

too high, also as expected. The overall picture of $\mathrm{SR}$ in the MV model discussed above qualitatively resembles that for the Ising model on SF networks with $\gamma \leq 3[28]$.

Concerning the MV model on strongly heterogeneous SF networks, the cases of networks with $2.5<\gamma \leq 3$ and with $2<\gamma<2.5$ must be distinguished. In the former case, according to the MFA, in the absence of the periodic signal the model still undergoes FM transition at a critical value $q=q_{c}<1 / 2$. The curves SPA vs. $q$ obtained from MC simulations still exhibit a single maximum typical of SR, independently of whether the SF network was generated from the CM or the UCM. This result is confirmed by theoretical analysis which is in particular based on the MFA, although quantitative agreement with results of MC simulation is deteriorated (not shown). Situation is more complex in the case of the MV model on SF networks with $2<\gamma<2.5$. In Figs. 3 and 4, theoretical predictions from the MFA and LRT for the model on SF network with $\gamma=2.25$ are summarized and compared with results of MC simulations. Results for the models on SF network with fully developed 
heterogeneity obtained from the CM (Fig. 3) as well as on the uncorrelated SF network obtained from the UCM (Fig. 4) are shown. According to the $\mathrm{MFA}$, in both cases - in the absence of the periodic signal - the MV model undergoes FM transition at a crossover value $q=q_{c}^{*}(N)$ which approaches $1 / 2$ in the thermodynamic limit. Also, in both cases and for a broad range of frequencies $\omega_{0}$, the curves SPA vs. $q$ obtained from MC simulations exhibit a single maximum indicating occurrence of SR, although for small $\omega_{0}$, in particular in the model on network generated from the CM, this maximum is accompanied with a sort of a hump. The hump is due to a slow increase of the SPA with $q$ in the FM phase. No additional maxima of the SPA were found in MC simulations, thus SMR was not observed. This is in contrast with the Ising model on SF networks with $2<\gamma<3$ (such that the crossover temperature for the FM transition diverges in the thermodynamic limit) generated from the $\mathrm{CM}$ in which SMR was observed in MC simulations [28].

In the case of the MV model on SF network with $\gamma=2.25$ generated from the CM model, even a qualitative agreement between the curves SPA vs. $q$ evaluated from the MFA and LRT and obtained from MC simulations is not satisfactory and, moreover, it deteriorates with approaching the adiabatic limit $\omega_{0} \rightarrow 0$ (Fig. 3). Both theoretical approximations predict that for small frequencies of the periodic signal, the SPA exhibits a sharp maximum close to or at $q=q_{c}^{*}(N)$ (see Fig. 3b, 3c) as well as a broad maximum at $q<q_{c}^{*}(N)$, in the FM phase. The occurrence of the two maxima of the SPA is a signature of SMR which is a finite-size effect which can be deduced from the location of the sharp peak of the SPA at the crossover value $q=q_{c}^{*}(N)$. These theoretical predictions are similar to those for the Ising model on the SF networks with $2<\gamma<3$ and fully developed heterogeneity [28] but for the MV model they are in contradiction with results of $\mathrm{MC}$ simulations. In the case of the MV model on the SF network with $\gamma=2.25$ generated from the UCM model, agreement between the curves SPA vs. $q$ evaluated from the MFA and LRT and obtained from MC simulations is better (see Fig. 4). Both theoretical approximations predict that for the whole range of the studied frequencies $\omega_{0}$, the SPA exhibits only one maximum, i.e, there is no SMR in accordance with results of MC simulations. Moreover, in the adiabatic limit $\omega_{0} \rightarrow 0$, the curve SPA vs. $q$ evaluated from the MFA using (5) and (6), (20) approaches the curve obtained from MC simulations in a similar manner as for the MV model on weakly heterogeneous SF networks (see Figs. 1 and 2), although the predicted values of the SPA are too low. It should be noted that the maxima of the SPA in Figs. 3 and 4, obtained both from theoretical predictions and MC simulations, are much lower than those in Figs. 1 and 2 and the maxima for the MV model on the SF networks generated from the CM (see Fig. 3)
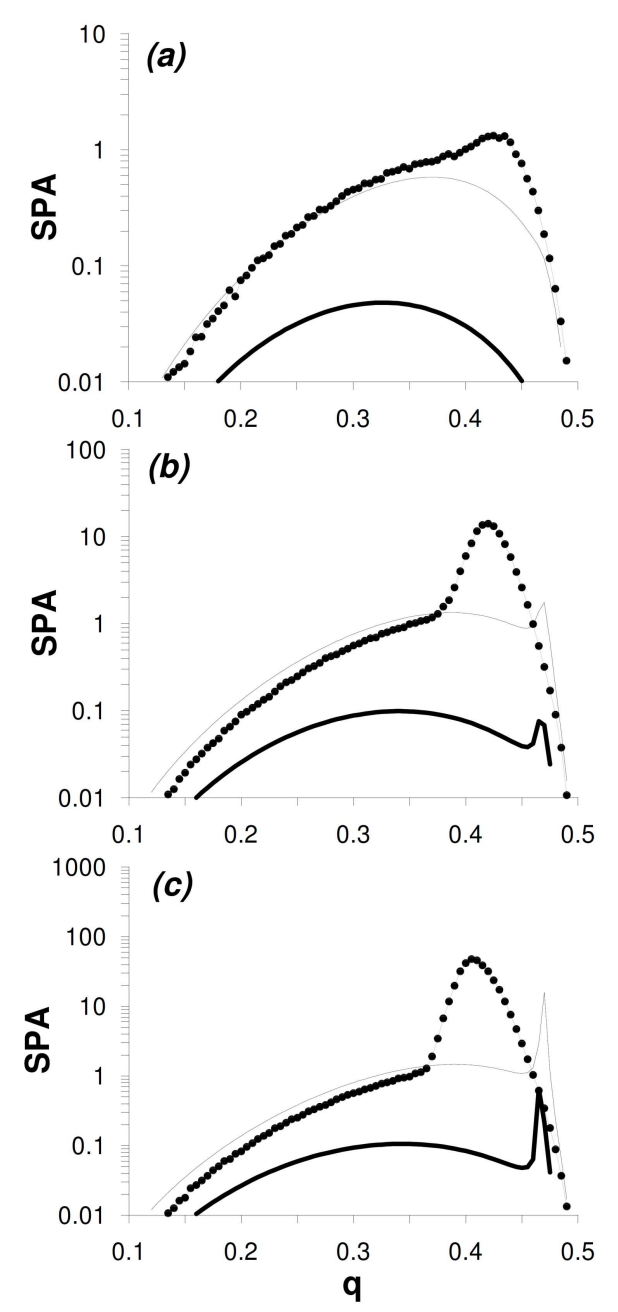

Fig. 3. SPA vs. $q$ for the MV model on an SF network obtained from the CM with $N=64000$, $k_{\text {min }}=5, \gamma=2.25$, for the periodic signal with $A=0.01$ and (a) $\omega_{0}=2 \pi / 8$, (b) $\omega_{0}=2 \pi / 32$, (c) $\omega_{0}=2 \pi / 128$. Symbols - results from MC simulations, gray solid lines are guides to the eyes, thick solid lines - results from numerical solution of the appropriate MF equations (20) and from (5) and (6), thin solid lines - results of the LRT, (27)-(32).

are lower and occur at higher values of $q$ than for the model on SF networks generated from the UCM (see Fig. 4). For small $\omega_{0}$, when the (possibly sharp) maxima are located close to $q=q_{c}^{*}(N)$, this confirms predictions of the LRT that in the case of the MV model on SF networks with fully developed heterogeneity the maxima of the SPA decrease and their location approaches $q=1 / 2$ faster with $N \rightarrow \infty$ than in the case of the model on the corresponding uncorrelated SF networks.

The comparison between Figs. 3 and 4 suggests that an important source of disagreement between theoretical predictions from the MFA or LRT and results of $\mathrm{MC}$ simulations concerning $\mathrm{SR}$ in the MV model on SF networks with $2<\gamma<2.5$ are correlations between degrees of nodes present in the network generated from the CM. In particular, 


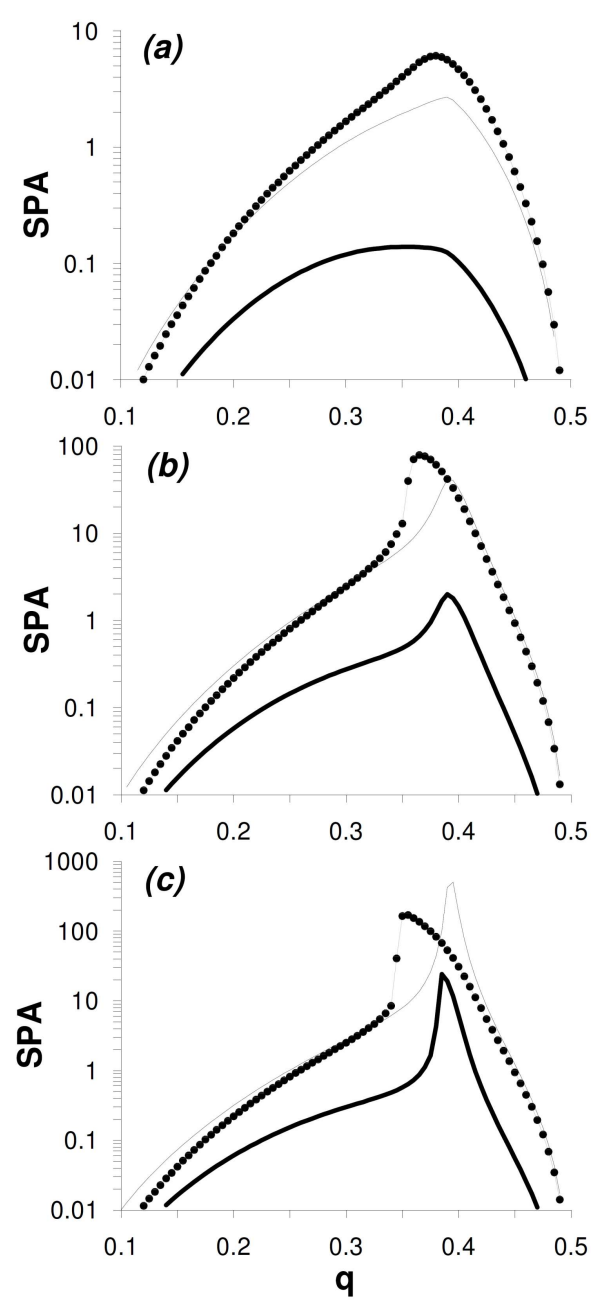

Fig. 4. As in Fig. 3 but for an SF network obtained from the UCM.

this results in overestimation of the crossover value $q_{c}^{*}(N)$ and hence in a shift of the sharp maximum of the SPA toward higher values of $q$ for small frequencies $\omega_{0}$. As the above-mentioned correlations are not present in the network generated from the $\mathrm{UCM}$, the agreement between theory and MC simulations is much improved. However, other factors can also contribute to the differences seen in Fig. 3. First, it is difficult to generate SF networks with small $\gamma$ and fully developed heterogeneity from the CM: nodes with very high degrees occur relatively seldom and even for a large but finite number of nodes $N$, the exponent $\gamma$ estimated from the numerical degree distribution $P(k)$ can be higher than expected from the model. According to the estimations from the LRT, this should correspond to a lower maximum degree $k_{\max }(N)$, lower crossover value $q_{c}^{*}(N)$ as well as a higher maximum of the SPA for low frequencies of the periodic signal $\omega_{0}$, i.e., $\left.\mathrm{SPA}\right|_{q=q_{c}^{*}(N)}$. Besides, agreement between the curves SPA vs. $q$ for low $\omega_{0}$ evaluated from the MFA using (5)-(20) and obtained from MC simulations deteriorates with decreasing exponent $\gamma$ even if correlations between degrees of nodes are not present in the SF network (Figs. 1c, 2c, 4c). This suggests that the relaxation of the MV model toward equilibrium at $q \neq q_{c}^{*}(N)$ also takes place more and more slowly with increasing $\gamma$ so that the adiabatic limit in which the predictions of the MFA should be more correct is not easily reached even for small $\omega_{0}$ accessible in simulations.

\section{Summary and conclusions}

SR in the MV model on SF networks with different heterogeneity and correlations between degrees of nodes, generated from the CM or UCM, was investigated by means of $\mathrm{MC}$ simulations and theoretically using heterogeneous MFA and LRT. In MC simulations for a broad range of frequencies of a weak external periodic signal, maxima of the SPA as a function of $q$, the parameter controlling the degree of stochasticity in the model, were observed which confirms the occurrence of SR. Theoretical predictions, particularly those based on direct application of equation for the time-dependence of the order parameter derived in the MFA, in the adiabatic limit of slowly varying periodic signal, shows a satisfactory quantitative agreement with results of MC simulations for the model on weakly heterogeneous SF networks or on heterogeneous uncorrelated SF networks. In the case of the MV model on strongly heterogeneous SF networks, structural SMR is predicted theoretically which, however, is not observed in MC simulations. This quantitative difference between predictions of the MFA and LRT and results of MC simulations may be due to strong correlations between degrees of nodes of SF networks with fully developed heterogeneity which makes the MFA derived for uncorrelated networks incorrect.

The absence of structural SMR in the nonequilibrium MV model on heterogeneous SF networks is in contrast with its occurrence in the related equilibrium Ising model. Nevertheless, from the point of view of modeling opinion formation, it is worth noting that the response of the MV model to a weak external signal can be maximized for optimum non-zero degree of internal (social) noise. In particular, it was shown in this paper that such an optimum response due to SR can occur in the MV model on SF networks which are often used as models for networks of social and economic interactions.

\section{References}

[1] M.J. Oliveira, J. Stat. Phys. 66, 273 (1992).

[2] C. Castellano, S. Fortunato, V. Loreto, Rev. Mod. Phys. 81, 591 (2009).

[3] M.J. Oliveira, J.F.F. Mendes, M.A. Santos, J. Phys. A Math. Gen. 26, 2317 (1993).

[4] A.L. Acu na-Lara, F. Sastre, Phys. Rev. E 86, 041123 (2012). 
[5] J. Yang, I. Kim, W. Kwak, Phys. Rev. E 77, 051122 (2008).

[6] L.F.C. Pereira, F.G. Brady Moreira, Phys. Rev. E 71, 016123 (2005).

[7] P.R.A. Campos, V.M. de Oliveira, F.G. Brady Moreira, Phys. Rev. E 67, 026104 (2003).

[8] T.E. Stone, S.R. McKay, Physica A 419, 437 (2015).

[9] F.W.S. Lima, Int. J. Mod. Phys. C 17, 1257 (2006).

[10] F.W.S. Lima, Commun. Comput. Phys. 2, 358 (2007).

[11] H. Chen, Ch. Shen, G. He, H. Zhang, Z. Hou, Phys. Rev. E 91, 022816 (2015).

[12] U. Yu, Phys. Rev. E 95, 012101 (2017).

[13] H. Feng, Ch. Han-Shuang, S. ChuanSheng, Chin. Phys. Lett. 32, 118902 (2015).

[14] A. Krawiecki, T. Gradowski, G. Siudem, Acta Phys. Pol. A 133, 1433 (2018).

[15] H. Chen, Ch. Shen, H. Zhang, G. Li, Z. Hou, J. Kurths, Phys. Rev. E 95, 042304 (2017).

[16] P.E. Harunari, M.M. de Oliveira, C.E. Fiore, Phys. Rev. E 96, 042305 (2017).

[17] A. Krawiecki, Int. J. Mod. Phys. B 29, 1750214 (2017).

[18] R. Benzi, A. Sutera, A. Vulpiani, J. Phys. A 14, L453 (1981).

[19] L. Gammaitoni, P. Hänggi, P. Jung, F. Marchesoni, Rev. Mod. Phys. 70, 223 (1998).

[20] M.D. McDonell, N.G. Stocks, C.E.M. Pearce, D. Abbott, Stochastic Resonance. From Suprathreshold Stochastic Resonance to Stochastic Signal Quantization, Cambridge University Press, Cambridge 2008.

[21] Z. Néda, Phys. Rev. E 51, 5315 (1995).

[22] J.J. Brey, A. Prados, Phys. Lett. A 216 , 240 (1996).

[23] U. Siewert, L. Schimansky-Geier, Phys. Rev. E 58, 2843 (1998).
[24] K.-T. Leung, Z. Néda, Phys. Lett. A 246, 505 (1998).

[25] K.-T. Leung, Z. Néda, Phys. Rev. E 59, 2730 (1999).

[26] B.J. Kim, P. Minnhagen, H.J. Kim, M.Y. Choi, G.S. Jeon, Europhys. Lett. 56, 333 (2001).

[27] H. Hong, B.J. Kim, M.Y. Choi, Phys. Rev. E 66, 011107 (2002).

[28] A. Krawiecki, Eur. Phys. J. B 69, 81 (2009).

[29] J.F. Lindner, B.K. Meadows, W.L. Ditto, M.E. Inchiosa, A.R. Bulsara, Phys. Rev. Lett. 75, 3 (1995).

[30] M. Gosak, M. Perc, S. Kralj, Eur. Phys. J. B 80, 519 (2011).

[31] H. Yu, X. Guo, J. Wang, Commun. Nonlin. Sci. Numer. Simulat. 42, 532 (2017).

[32] A.-L. Barabási, R. Albert, Science 286, 509 (1999).

[33] R. Albert, A.-L. Barabási, Rev. Mod. Phys. 74, 47 (2002).

[34] J.M.G. Vilar, J.M. Rubí, Phys. Rev. Lett. 78, 2882 (1997).

[35] J.M.G. Vilar, J.M. Rubí, Physica A 264, 1 (1999).

[36] J.F. Mejias, J.J. Torres, PLOS One 6, e17255 (2011).

[37] G. Pinamonti, J. Marro, J.J. Torres, PLOS One 7, e51170 (2012).

[38] J.J. Torres, I. Elices, J. Marro, PLOS One 10, e0121156 (2015).

[39] H. Li, X. Sun, J. Xiao, Chaos 28, 043113 (2018).

[40] M. Kaim, A. Krawiecki, Phys. Lett. A 374, 4814 (2010).

[41] S.N. Dorogovtsev, A.V. Goltsev, J.F.F. Mendes, Phys. Rev. E 66, 016104 (2002).

[42] M.E.J. Newman, in: Handbook of Graphs and Networks: From the Genome to the Internet, Eds. S. Bornholdt, H.G. Schuster, Wiley-VCH, Berlin 2003, p. 35.

[43] M. Catanzaro, M. Boguńá, R. PastorSatorras, Phys. Rev. E 71, 027103 (2005). 